\title{
Calidad de la alimentación y estado nutricional en estudiantes universitarios de 11 regiones de Chile
}

\author{
RINAT RATNER G. ${ }^{1, a, b}$, PAULINA HERNÁNDEZ J. ${ }^{1, a}$, \\ JORGE MARTEL A. ${ }^{1, \mathrm{c}}$, EDUARDO ATALAH S. ${ }^{2}$
}

\section{Food quality and nutritional status in university students of eleven Chilean regions}

\begin{abstract}
Background: The Chilean population has inadequate lifestyles and high prevalence of chronic diseases. Aim: To analyze eating behaviors, nutritional status and history of previous diseases, in students of higher education. Material and Methods: Cross-sectional study in students of 54 higher education centers across the country. They answered a survey about dietary habits, physical activity, smoking, previous diseases and opinion of their nutritional condition. Weight and height were measured under standardized conditions and nutritional status classified according to body mass index. Results: We studied 6,823 students aged 17 to 29 years. Forty seven percent did not have breakfast and 35\% did not have lunch every day. A low proportion had a daily consumption of vegetables (51.2\%), fruits (39.4\%) and dairy products (57.5\%). There was a high frequency of soft drinks, chips, cakes and sweets consumption. Seventy six percent were sedentary, $40.3 \%$ smokers and $27.4 \%$ overweight or obese. The latter had a significantly higher frequency of diabetes, hypertension and hypercholesterolemia. There was a poor agreement between actual nutritional status and self-perception, especially in males (Kappa index 0.38). Recipients of a food scholarship provided by the Ministry of Education ate lunch usually with a higher frequency $(p<0.05)$. Conclusions: A high prevalence of inadequate eating and physical activity patterns in these young subjects with good educational level was observed. The food scholarship has some positive effects, although differences in socioeconomic levels limited comparisons.
\end{abstract}

(Rev Med Chile 2012; 140: 1571-1579).

Key words: Adolescent; Chile; Eating; Feeding behavior; Obesity.

'Sodexo Soluciones de
Motivación, Santiago,
Chile.
Departamento de
Nutrición, Facultad de
Medicina, Universidad de
Chile. Santiago, Chile.
aNutricionista.
bMagíster en Nutrición
Clínica.
'Ingeniero Comercial.
Conflictos de intereses:
Estudio financiado por la
empresa Sodexo Soluciones
de Motivación, cuyos
profesionales participaron
en el diseño, análisis e
interpretación de los datos.
Recibido el 27 de febrero
de 2012, aceptado el 7 de
agosto de 2012.
Correspondencia a:
Dr. Eduardo Atalah S.
Departamento de
Nutrición, Facultad de
Medicina, Universidad
de Chile. Independencia
1027, Santiago de Chile.
Teléfono: 9786241.
E-mail: eatalah@med.
uchile.cl

$\mathrm{D}$ iversos estudios nacionales han demostrado la mala calidad de la alimentación de la población chilena a través de todo su ciclo vital. Los principales problemas alimentarios se vinculan con bajos consumos de frutas, verduras, leguminosas, pescados y lácteos y alto consumo de alimentos industrializados, que generalmente contribuyen a un aporte excesivo de calorías, grasas, azúcar y sal ${ }^{1-4}$. Existe también una alta prevalencia de tabaquismo, obesidad y enfermedades crónicas vinculadas con los estilos de vida ${ }^{2,5-7}$, al igual que en muchos otros países ${ }^{8-12}$.
La preocupación por el sedentarismo y la alimentación se basa en sus efectos directos en la calidad de vida de las personas, por su fuerte asociación con enfermedades cardiovasculares, diabetes mellitus tipo 2, hipertensión arterial, enfermedades osteoarticulares y algunos tipos de cáncer. La obesidad puede llegar a reducir la esperanza de vida hasta en diez años y representa una elevada carga económica para la sociedad ${ }^{13-16}$.

Aun cuando se han desarrollado diversas estrategias para mejorar las conductas alimentarias y 
estilos de vida de la población chilena, los resultados obtenidos hasta la fecha han sido limitados ${ }^{17-19}$ y la prevalencia de obesidad ha continuado en aumento en niños, escolares y adultos.

La información disponible en el país sobre alimentación y estilos de vida en adolescentes y adultos jóvenes es limitada. La Encuesta Nacional de Salud del año 2010 incluyó 812 jóvenes de 15 a 24 años, donde se observó 10,9\% de obesidad, $46 \%$ de fumadores actuales, $79,3 \%$ de sedentarismo y que sólo $13 \%$ cumplía con las recomendaciones de consumo de verduras y frutas del Ministerio de Salud $^{6}$. Un estudio en 449 estudiantes universitarios de Santiago mostró bajos niveles de actividad física y de consumo de verduras y frutas, asociados a una baja disposición al cambio (etapa de contemplación en el modelo de Prochaska). La principal barrera descrita para realizar mayor actividad física fue la limitada disponibilidad de tiempo (68\%) y para un mayor consumo de verduras y frutas "flojera" para preparar ensaladas, falta de tiempo, olvido y el hecho que estos alimentos no quitan el hambre ${ }^{20}$.

El Ministerio de Educación implementa desde hace algunos años una beca de alimentación para la educación superior (Beca BAES), cuyo objetivo es apoyar la permanencia y egreso de la Educación Superior a estudiantes vulnerables. Consiste en un subsidio de alimentación ( $\$ 1.300$ pesos diarios de marzo a diciembre de cada año), entregado a través de una tarjeta electrónica de canje, que es utilizado en una red de locales especializados en la venta de productos alimenticios y supermercados. La Beca BAES está dirigida a estudiantes que ingresan a primer año de Educación Superior y que pertenecen a los quintiles de ingreso más vulnerables (I y II), que reciben Créditos Universitarios (con Garantía del Estado o Fondo Solidario) o reciben diferentes Becas de Arancel (Bicentenario, Nuevo Milenio, Juan Gómez Millas, de Excelencia Académica, Puntaje PSU, Vocación de Profesor o para hijos de Profesionales de la Educación) ${ }^{21}$.

El objetivo de este estudio es analizar la calidad de la alimentación, actividad física y antecedentes de patologías previas en estudiantes de educación superior en función de su estado nutricional y de su participación en las Becas BAES. Sus resultados pueden contribuir a conocer mejor las brechas existentes en la calidad de la alimentación en esta población y a definir nuevas estrategias de intervención.

\section{Material y Métodos}

El estudio se realizó en 54 casas de estudios superiores en 11 regiones del país, donde las becas de alimentación BAES son gestionadas por la Empresa "Sodexo Soluciones de Motivación". En cada una de las instituciones estudiadas se solicitó la aprobación de la autoridad universitaria correspondiente, para instalar estaciones móviles de evaluación del estado nutricional. El stand fue emplazado durante uno a tres días en cada casa de estudios y se ofreció aplicar la encuesta y realizar las mediciones antropométricas a los estudiantes que voluntariamente decidieron participar.

Para estimar el tamaño de la muestra se consideró una diferencia de 5 puntos porcentuales en la frecuencia de consumo semanal de los principales tiempos de comidas y grupos de alimentos en función del estado nutricional y participación en las becas BAES, un nivel de significación 0,05 y poder de $80 \%$, lo que determina la necesidad de estudiar 1.511 personas en cada grupo.

El trabajo de terreno fue realizado durante los años 2010 y 2011. En una primera etapa se aplicó una encuesta que incluyó información sobre hábitos alimentarios, actividad física y tabaquismo:

- Frecuencia de consumo semanal de los principales tiempos de comida (desayuno, almuerzo, once, cena, colación).

- Frecuencia de consumo diaria o semanal de algunos grupos de alimentos de importancia en salud pública: frituras, bebida gaseosas, pasteles/ galletas/tortas/ dulces o chocolates, frutas, verduras, pescados, leguminosas y leche o derivados. Para cada uno de estos grupos de alimentos se consideraron las siguientes alternativas: $3 \mathrm{o}$ más veces al día, 2 veces al día, 1 vez al día, 4 a 6 veces a la semana, 2 a 3 veces a la semana, una vez a la semana y ocasional o nunca.

- Práctica de actividad física o deporte durante 30 min o más: 3 o más veces por semana; 1 a 2 veces por semana; menos de 4 veces al mes; no practica u ocasional.

- Antecedentes de haber fumado el último mes.

- Autopercepción del estado nutricional en relación a figuras de mujeres y hombres con bajo peso, normal, sobrepeso y obeso.

- Antecedentes de que se le haya diagnosticado previamente hipertensión arterial, diabetes, hipercolesterolemia, depresión y enfermedades respiratorias. 
- Participación en la Beca de Alimentación para Educación Superior (Beca BAES), dato que se obtuvo sólo durante el año 2011.

Una vez completada la encuesta se midió peso y talla por nutricionistas, siguiendo técnicas estandarizadas. El peso se obtuvo con ropa ligera y descalzo con balanza portátil marca SECA, con precisión de $100 \mathrm{~g}$, calibrada periódicamente. La talla se midió con tallímetro portátil marca SECA con $0,1 \mathrm{~cm}$ de precisión. A partir del peso y la talla se calculó el índice de masa corporal $\left(\mathrm{kg} / \mathrm{m}^{2}\right)$ y se clasificó el estado nutricional según la norma OMS: enflaquecido $<18,5 \mathrm{~kg} / \mathrm{m}^{2}$ ), normopeso $18,5-24,9 \mathrm{~kg} / \mathrm{m}^{2}$, sobrepeso $25,0-29,9 \mathrm{~kg} / \mathrm{m}^{2}$ y obesos $\geq 30 \mathrm{~kg} / \mathrm{m}^{2}$.

\section{Análisis estadísticos}

Los datos fueron analizados con el programa STATA11.0. Se analizaron promedio y desviación estándar en las variables continuas con distribución normal y distribución de frecuencia en las variables categóricas. Para la comparación entre los grupos se utilizó prueba de ANOVA para muestras independientes en las variables continuas y $\chi^{2}$ en las variables categóricas, considerando significativo un valor de $\mathrm{p}<0,05$. Para comparar la autopercepción del estado nutricional con el diagnóstico real según mediciones antropométricas se utilizó el índice Kappa, considerándose una buena concordancia un valor $\geq 0,80$.

\section{Resultados}

Se estudiaron 6.823 estudiantes, con mayor proporción de mujeres $(63,3 \%)$ cuya edad, características antropométricas y estado nutricional se presentan en la Tabla 1. Se trata de adultos jóvenes $(21,3 \pm 2,5$ años), con un IMC promedio cercano al límite superior de la normalidad, especialmente en varones. La mayor parte de la muestra presentó un estado nutricional normal, aunque ya existe $27,4 \%$ de sobrepeso u obesidad, con una proporción significativamente mayor en varones. Un análisis similar en función de la participación en las Becas BAES no demostró diferencias en el estado nutricional.

La frecuencia de consumo de los distintos tiempos de comida diaria se presenta en la Tabla 2. Destaca que menos de la mitad de los encuestados consume diariamente desayuno, que cerca de un tercio de los jóvenes no almuerza todos los días y que la frecuencia de consumo habitual de la cena es muy baja. Existen diferencias por estado nutricional, con mayor frecuencia de consumo de desayuno, once y comidas en los estudiantes con peso normal, lo que determina un mayor fraccionamiento de la alimentación. Los beneficiarios de la Beca BAES presentan conductas similares al grupo no intervenido, aunque el consumo diario de almuerzo es algo mayor $(\mathrm{p}<0,05)$ y existe una tendencia a una mayor frecuencia de consumo diario de desayuno (p 0,069), a pesar

Tabla 1. Características antropométricas de la población estudiada según sexo y participación en el programa de becas alimentarias de la JUNAEB

\begin{tabular}{|c|c|c|c|c|c|c|}
\hline Variable & $\begin{array}{c}\text { Mujeres } \\
n=4.317 \\
X \pm D E\end{array}$ & $\begin{array}{c}\text { Hombres } \\
n=2.506 \\
X \pm D E\end{array}$ & $\mathbf{p}$ & $\begin{array}{c}\text { Beca BAES } \\
n=1.833 \\
x \pm D E\end{array}$ & $\begin{array}{c}\text { Sin Beca } \\
n=1.474 \\
x \pm D E\end{array}$ & $\mathbf{p}$ \\
\hline Edad (años) & $21,2 \pm 2,5$ & $21,4 \pm 2,5$ & $<0,01$ & $21,6 \pm 2,3$ & $20,9 \pm 2,7$ & $<0,01$ \\
\hline Peso $(\mathrm{Kg})$ & $59,3 \pm 9,6$ & $72,7 \pm 11,3$ & $<0,001$ & $64,8 \pm 8,9$ & $63,6 \pm 8,8$ & $<0,001$ \\
\hline Talla (cm) & $160,0 \pm 6,0$ & $173,1 \pm 6,8$ & $<0,001$ & $165,4 \pm 6,0$ & $163,9 \pm 6,8$ & $<0,001$ \\
\hline IMC & $23,2 \pm 3,5$ & $24,2 \pm 3,2$ & $<0,001$ & $23,5 \pm 3,4$ & $23,6 \pm 3,5$ & NS \\
\hline Estado nutricional & $\%$ & $\%$ & $p$ & $\%$ & $\%$ & $p$ \\
\hline Bajo peso & 3,9 & 1,5 & & 3,4 & 2,5 & \\
\hline Normal & 72,2 & 65,1 & & 69,8 & 70,4 & \\
\hline Sobrepeso & 19,8 & 27,9 & & 22,3 & 22,5 & \\
\hline Obesidad & 4,1 & 5,5 & $<0,001$ & 4,5 & 4,6 & NS \\
\hline
\end{tabular}

IMC: Índice de masa corporal. 
Calidad de alimentación y estado nutricional en universitarios - R. Ratner et al

Tabla 2. Frecuencia de consumo semanal de los distintos tiempos de comida, según estado nutricional y participación en las becas de alimentación de JUNAEB

\begin{tabular}{|c|c|c|c|c|c|c|}
\hline \multirow[t]{2}{*}{ Comida } & \multicolumn{3}{|c|}{ Estado nutricional } & \multicolumn{3}{|c|}{ Beca BAES } \\
\hline & $\begin{array}{c}\text { Bajo peso/ } \\
\text { normal } \\
\%\end{array}$ & $\begin{array}{c}\text { Sobrep./ } \\
\text { obeso } \\
\%\end{array}$ & $\mathbf{p}$ & $\begin{array}{c}\text { Con Beca } \\
\%\end{array}$ & $\begin{array}{c}\text { Sin Beca } \\
\%\end{array}$ & $\mathbf{p}$ \\
\hline \multicolumn{7}{|l|}{ Desayuno } \\
\hline 0 a 2 días/sem & 15,5 & 22,1 & & 16,9 & 17,6 & \\
\hline 3 a 6 días/sem & 40,0 & 40,9 & & 39,6 & 43,0 & \\
\hline Diariamente & 44,5 & 37,0 & $<0,01$ & 43,5 & 39,4 & 0,069 \\
\hline \multicolumn{7}{|l|}{ Almuerzo } \\
\hline 0 a 2 días/sem & 3,6 & 4,5 & & 4,8 & 3,2 & \\
\hline 3 a 6 días/sem & 31,7 & 30,3 & & 29,8 & 32,9 & \\
\hline Diariamente & 64,7 & 65,2 & NS & 65,4 & 63,9 & $<0,05$ \\
\hline \multicolumn{7}{|l|}{ Once* } \\
\hline 0 a 2 días/sem & 7,4 & 9,1 & & 7,8 & 6,3 & \\
\hline 3 a 6 días/sem & 27,5 & 28,9 & & 29,4 & 28,9 & \\
\hline Diariamente & 65,1 & 62,2 & $<0,05$ & 62,8 & 64,8 & NS \\
\hline \multicolumn{7}{|l|}{ Comida } \\
\hline 0 a 2 días/sem & 54,1 & 58,2 & & 51,3 & 51,0 & \\
\hline 3 a 6 días/sem & 24,7 & 23,0 & & 25,4 & 28,0 & \\
\hline Diariamente & 21,2 & 18,8 & $<0,05$ & 23,3 & 21,0 & NS \\
\hline \multicolumn{7}{|l|}{ Colación } \\
\hline 0 a 2 días/sem & 19,7 & 25,2 & & 17,8 & 18,0 & \\
\hline 3 a 6 días/sem & 42,9 & 40,6 & & 42,2 & 43,9 & \\
\hline Diariamente & 37,4 & 34,2 & $<0,01$ & 40,0 & 38,1 & NS \\
\hline
\end{tabular}

*Once $=$ colación a media tarde.

de que se trata de un grupo de menor nivel socioeconómico.

El consumo de los principales grupos de alimentos se aleja bastante de las Guías Alimentarias del Ministerio de Salud (Tabla 3). Se observó alta frecuencia de consumo de pasteles, galletas y dulces, bebidas gaseosas y frituras y baja frecuencia de consumo diario de frutas, verduras y lácteos. Destaca también que cerca de la mitad de la muestra no consumía pescado y un tercio no consumía leguminosas o en forma ocasional. Aun cuando existen algunas diferencias por estado nutricional, estas no son muy acentuadas, lo que refleja que las inadecuadas conductas alimentarias son independientes del estado nutricional. El mismo análisis en el grupo con o sin Beca alimentaria no muestra grandes diferencias, lo que sugiere que no existe un impacto positivo en la alimentación, aunque tampoco habría una externalidad negativa, reflejada en un mayor consumo de alimentos no saludables.
El tabaquismo, niveles de actividad física y los antecedentes de patologías previas se presentan en la Tabla 4. Se observó una alta proporción de fumadores, especialmente en estudiantes con sobrepeso y bajos niveles de actividad física, en ambos grupos. Una alta proporción de la muestra tenía antecedentes de patologías previas, especialmente depresión y patologías respiratorias. Aunque los antecedentes de diabetes, hipertensión e hipercolesterolemia son relativamente bajos, existe un aumento significativo de la prevalencia en el grupo con exceso de peso. Destaca también un aumento importante de casi todos los factores de riesgo y de los antecedentes de patologías previas en un corto período de tiempo.

La comparación entre el diagnóstico nutricional real y la autopercepción mostró una muy baja concordancia (Tabla 5). Las principales diferencias se deben a una subestimación del peso en más de $70 \%$ de los estudiantes obesos y una sobreestima- 
Calidad de alimentación y estado nutricional en universitarios - R. Ratner et al

Tabla 3. Frecuencia de consumo semanal de algunos grupos de alimentos, según estado nutricional y participación en las becas de alimentación de JUNAEB

\begin{tabular}{|c|c|c|c|c|c|c|}
\hline \multirow[t]{2}{*}{ Alimentos } & \multicolumn{3}{|c|}{ Estado nutricional } & \multicolumn{3}{|c|}{ Beca BAES } \\
\hline & $\begin{array}{c}\text { B. Peso/ } \\
\text { Normal } \\
\%\end{array}$ & $\begin{array}{c}\text { Sobrep./ } \\
\text { Obeso } \\
\%\end{array}$ & $\mathbf{p}$ & $\begin{array}{c}\text { Con Beca } \\
\%\end{array}$ & $\begin{array}{c}\text { Sin Beca } \\
\%\end{array}$ & $\mathbf{p}$ \\
\hline \multicolumn{7}{|l|}{ Frituras } \\
\hline Todos los días & 15,8 & 16,0 & & 17,4 & 16,4 & \\
\hline Algunos días/sem & 60,8 & 62,5 & & 59,3 & 61,2 & \\
\hline Ocasional/nunca & 23,4 & 21,5 & NS & 23,3 & 22,4 & NS \\
\hline \multicolumn{7}{|l|}{ Bebidas gaseosas } \\
\hline Todos los días & 29,1 & 31,3 & & 35,0 & 31,7 & \\
\hline Algunos días/sem & 48,8 & 50,7 & & 45,2 & 48,9 & \\
\hline Ocasional/nunca & 22,1 & 18,0 & $<0,001$ & 19,8 & 19,4 & 0,08 \\
\hline \multicolumn{7}{|c|}{ Pasteles/dulces/chocol. } \\
\hline Todos los días & 66,9 & 59,9 & & 62,8 & 63,6 & \\
\hline Algunos días/sem & 28,9 & 32,7 & & 30,6 & 31,3 & \\
\hline Ocasional/nunca & 4,2 & 7,4 & $<0,001$ & 6,6 & 5,1 & NS \\
\hline \multicolumn{7}{|l|}{ Frutas } \\
\hline Todos los días & 40,0 & 38,0 & & 40,7 & 38,0 & \\
\hline Algunos días/sem & 47,4 & 48,6 & & 45,2 & 48,4 & \\
\hline Ocasional/nunca & 12,6 & 13,4 & NS & 14,1 & 13,6 & NS \\
\hline \multicolumn{7}{|l|}{ Verduras } \\
\hline Todos los días & 51,7 & 51,5 & & 51,3 & 52,2 & \\
\hline Algunos días/sem & 43,1 & 43,9 & & 43,3 & 42,9 & \\
\hline Ocasional/nunca & 5,2 & 4,6 & NS & 5,4 & 4,9 & NS \\
\hline \multicolumn{7}{|l|}{ Lácteos } \\
\hline Todos los días & 59,2 & 53,0 & & 57,7 & 56,2 & \\
\hline Algunos días/sem & 33,8 & 38,0 & & 33,9 & 35,6 & \\
\hline Ocasional/nunca & 7,0 & 9,0 & $<0,001$ & 8,4 & 8,2 & NS \\
\hline \multicolumn{7}{|l|}{ Pescado } \\
\hline$\geq 2$ veces/sem & 13,7 & 15,2 & & 15,6 & 14,0 & \\
\hline Una vez/sem & 40,0 & 35,3 & & 38,2 & 35,8 & \\
\hline Ocasional/nunca & 49,3 & 49,5 & NS & 46,2 & 50,2 & 0,07 \\
\hline \multicolumn{7}{|l|}{ Leguminosas } \\
\hline$\geq 2$ veces/sem & 22,1 & 23,0 & & 24,6 & 21,3 & \\
\hline Una vez/sem & 46,0 & 45,4 & & 45,8 & 45,7 & \\
\hline Ocasional/nunca & 31,9 & 31,6 & NS & 29,6 & 33,0 & $<0,05$ \\
\hline
\end{tabular}


Tabla 4. Tabaquismo, actividad física y antecedentes de patologías según estado nutricional y edad

\begin{tabular}{|c|c|c|c|c|c|c|c|}
\hline \multirow[b]{2}{*}{ Variable } & \multicolumn{3}{|c|}{ Estado nutricional } & \multicolumn{4}{|c|}{ Edad años } \\
\hline & $\begin{array}{c}\text { B. Peso/ } \\
\text { normal } \\
\%\end{array}$ & $\begin{array}{c}\text { Sobrep./ } \\
\text { obeso } \\
\%\end{array}$ & $\mathbf{p}$ & $\begin{array}{c}\text { 17-19 } \\
\%\end{array}$ & $\begin{array}{c}20-22 \\
\%\end{array}$ & $\begin{array}{c}23-29 \\
\%\end{array}$ & $\mathbf{p}$ \\
\hline Fumador & 37,9 & 46,5 & $<0,001$ & 35,6 & 40,3 & 44,7 & $<0,001$ \\
\hline \multicolumn{8}{|l|}{ Actividad física } \\
\hline$\geq 3$ veces/sem & 24,5 & 24,6 & & 22,1 & 25,8 & 24,3 & \\
\hline 1 a 2 veces/sem & 29,0 & 30,4 & & 31,0 & 29,5 & 27,5 & \\
\hline Ocasional & 10,4 & 11,6 & & 12,8 & 10,5 & 9,5 & \\
\hline No realiza & 36,1 & 33,3 & NS & 34,1 & 34,2 & 38,7 & $<0,001$ \\
\hline Sobrep/obesidad & & & & 21,6 & 26,3 & 35,3 & $<0,001$ \\
\hline \multicolumn{8}{|l|}{ Antecedentes de: } \\
\hline Hipertensión arterial & 1,7 & 3,9 & $<0,001$ & 1,7 & 1,9 & 3,9 & $<0,001$ \\
\hline Diabetes & 1,7 & 3,9 & $<0,001$ & 2,1 & 2,3 & 2,4 & NS \\
\hline Hipercolesterolemia & 4,4 & 6,9 & $<0,001$ & 3,9 & 5,0 & 6,5 & $<0,005$ \\
\hline Respiratorias & 12,7 & 15,4 & $<0,05$ & 14,1 & 13,2 & 13,1 & NS \\
\hline Depresión & 12,4 & 16,5 & $<0,005$ & 9,3 & 13,8 & 17,7 & $<0,005$ \\
\hline
\end{tabular}

Tabla 5. Comparación del estado nutricional real y la auto percepción, según sexo

\begin{tabular}{|c|c|c|c|c|c|c|}
\hline \multirow[t]{2}{*}{ Estado nutricional } & \multicolumn{2}{|c|}{ Mujeres \% } & \multicolumn{2}{|c|}{ Hombre \% } & \multicolumn{2}{|c|}{ Total \% } \\
\hline & Sub-estima & Sobre-estima & Sub-estima & Sobre-estima & Sub-estima & Sobre-estima \\
\hline Bajo peso & - & 48,8 & - & 18,8 & - & 44,2 \\
\hline Normal & 3,8 & 16,9 & 16,8 & 9,8 & 8,1 & 14,5 \\
\hline Sobrepeso & 18,9 & 3,0 & 42,1 & 1,3 & 29,0 & 2,3 \\
\hline Obeso & 60,7 & - & 88,5 & - & 72,4 & - \\
\hline Concordancia \% & \multicolumn{2}{|c|}{76,3} & \multicolumn{2}{|c|}{65,5} & \multicolumn{2}{|c|}{72,5} \\
\hline Indice Kappa & \multicolumn{2}{|c|}{0,51} & \multicolumn{2}{|c|}{0,38} & \multicolumn{2}{|c|}{0,49} \\
\hline
\end{tabular}

ción en casi la mitad de los jóvenes de bajo peso. El comportamiento es significativamente diferente según sexo, con mayor sobreestimación del peso en las mujeres de bajo peso y peso normal y mayor subestimación en los varones con sobrepeso y obesos.

\section{Discusión}

El estudio muestra una alta prevalencia de conductas alimentarias inadecuadas, tabaquismo y sedentarismo en estudiantes de educación supe- rior, lo que se asocia con exceso de peso y antecedentes de algunas patologías crónicas asociadas a los estilos de vida. Ello es especialmente preocupante considerando la magnitud del problema, la precocidad con que se presenta y el incremento casi al doble de los factores de riesgo en el grupo con exceso de peso, de 23 años y más. También es alarmante el hecho de que se trata de estudiantes universitarios o de educación técnica superior, que podrían estar más informados sobre la importancia de una alimentación saludable y más dispuestos a asumirla, considerando sus múltiples beneficios en la salud. 
Destaca el hecho que menos de la mitad de los jóvenes desayuna todos los días, lo que puede afectar la capacidad de aprendizaje $e^{22,23}$ y puede determinar un mayor consumo de colaciones, generalmente de baja calidad nutricional y alta densidad energética ${ }^{24,25}$. El estudio demostró una menor frecuencia de comidas diarias en las personas con sobrepeso, una conducta negativa que se ha asociado a una mayor ingesta energética y mayor riesgo de obesidad en algunos estudios ${ }^{26-28}$.

La alta frecuencia de consumo semanal de alimentos poco saludables se asocia con una mayor ingesta energética, grasas, azúcar y sodio y mayor riesgo de obesidad, hipertensión arterial e hipercolesterolemia ${ }^{29}$. Por otra parte, el bajo consumo de frutas, verduras y lácteos se asocia con menor consumo de antioxidantes, fibra y calcio, lo que también tiene efectos negativos en la salud en relación al riesgo de cáncer y enfermedades cardiovasculares, entre varias otras ${ }^{30-33}$. Si ello se asocia a bajos niveles de actividad física y tabaquismo, no sorprende encontrar que a una fracción de la población estudiada ya se le ha diagnosticado alguna enfermedad crónica no transmisible, con un importante incremento en función del estado nutricional.

Llama la atención la alta frecuencia de antecedentes de problemas respiratorios, aun cuando ello es consistente con la Encuesta Nacional de Salud 2010, donde la sospecha de síntomas respiratorios crónicos (tosedor crónico, asma bronquial, disnea) afectó al $15,7 \%$ de la población de 15 a 24 años ${ }^{5}$. En este estudio no es posible diferenciar cual de los síntomas es más prevalente, ya que la pregunta era de tipo general, pero refleja la existencia de un importante problema de salud pública. Lo mismo sucede con la depresión, que habría sido diagnosticada en $14 \%$ de la muestra de universitarios estudiada, cifra similar a la que entrega la Encuesta Nacional de Salud, que mostró una prevalencia global de 21,7\% ${ }^{5}$.

Otro hecho destacable es la inadecuada percepción que los jóvenes tienen en relación a su estado nutricional real. Los problemas se relacionan fundamentalmente con una sobreestimación del peso en mujeres con peso normal y subestimación del peso en varones con exceso de peso. El 27,5\% de la muestra estudiada tuvo una percepción errónea de su estado nutricional, similar a lo observado en otro estudio en población adulta ${ }^{34}$, lo que determina que no se adopten las conductas alimentarias adecuadas que corresponderían a cada caso específico.

Aun cuando la Beca BAES no tiene un objetivo propiamente nutricional, es interesante evaluar sus potenciales externalidades positivas (mayor frecuencia de consumo de desayuno, almuerzo, verduras, frutas o lácteos, por ejemplo) o externalidades negativas en las conductas alimentarias o tabaquismo (mayor consumo de bebidas, golosinas, cigarrillos). El estudio no mostró diferencias importantes entre los dos grupos, aunque los beneficiarios de la beca pertenecen a los dos primeros quintiles de ingreso, lo que sin duda es una variable de confusión que puede afectar los resultados. Desde ese punto de vista, el hecho de que las conductas alimentarias fueran similares con los estudiantes de mayores ingresos o que existiera una mayor frecuencia de almuerzos diarios, podría considerarse un resultado positivo del programa.

La investigación tiene la fortaleza de haber obtenido información en un grupo poco estudiado, con datos obtenidos en 11 regiones del país y en 54 centros de educación superior, lo que sugiere una buena representatividad. Sin embargo, la muestra no fue aleatoria, sino más bien por decisión de los propios jóvenes, lo que limita la posibilidad de extrapolar sus resultados. De hecho llama la atención una baja prevalencia de obesidad, que representa la mitad de lo observado en la Encuesta Nacional de Salud 2010 en ese grupo de edad, lo que podría ser interpretado por auto exclusión de los jóvenes con mayor exceso de peso o con peores conductas alimentarias. A pesar de ello las conductas alimentarias observadas fueron relativamente similares en todos los grupos analizados, lo que permite suponer que los resultados tendrían cierta confiabilidad.

Los datos del estudio confirman los importantes desafíos que existen en salud pública en relación a la alimentación y estilos de vida y los escasos resultados obtenidos con las estrategias de prevención aplicadas hasta ahora (Estrategia Global Contra la Obesidad, Estrategia de intervención a través del ciclo vital, Cinco al día, Elige Vivir Sano, Escuelas Saludables, etc). Ello muestra la necesidad de implementar nuevas estrategias de intervención, incluyendo restricciones a la publicidad y comercialización de alimentos poco saludables en escolares, mayor promoción de la actividad física a nivel de escuelas y de comunidades, campañas educativas y la implementación 
Calidad de alimentación y estado nutricional en universitarios - R. Ratner et al

de un sistema de información nutricional en los alimentos industrializados de fácil comprensión para el consumidor. La nueva ley de alimentos, recientemente promulgada, puede ser un importante avance en ese sentido.

\section{Referencias}

1. Crovetto M, Uauy R. Cambios en el consumo aparente de nutrientes en el Gran Santiago 1988-1997 en hogares según ingreso y su probable relación con patrón de enfermedades crónicas no transmisibles. Rev Med Chile 2010; 138: 1091-108.

2. Ratner R, Sabal J, Hernández P, Romero D, Atalah E. Estilos de Vida y Estado Nutricional de trabajadores Chilenos de Empresas Públicas y Privadas de dos Regiones de Chile. Rev Med Chile 2008; 136: 1406-14.

3. Burrows R, Díaz E, Sciaraffia V, Gattas V, Montoya A, Lera L, Burrows R, et al. Hábitos de ingesta y actividad física en escolares, según tipo de establecimiento al que asisten. Rev Med Chile 2008; 136: 53-63.

4. Zacarías I, Rodríguez L, Lera L, Hill R, Domper A, González D. Consumo de verduras y frutas en Centros de Salud y supermercados, de la Región Metropolitana de Chile: Programa 5 al día. Rev Chil Nutr 2009; 36: 159-68.

5. Encuesta Nacional de Salud ENS 2009-2010. Disponible en: http://www.redsalud.gov.cl/portal/docs/page/ minsalcl/g_home/submenu_portada_2011/ens2010.pdf (Consultado el 18 de enero de 2012).

6. Vio F, Albala C, Kain J. Nutrition transition in Chile revisited: mid-term evaluation of obesity goals for the period 2000-2010. Public Health Nutr 2008; 11: 405-12.

7. Fagalde MP, del Solar JA, Guerrero M, Atalah E. Factores de riesgo de enfermedades crónicas no transmisibles en funcionarios de una empresa de servicios financieros de la Región Metropolitana. Rev Med Chile 2005; 919-28.

8. Faeh D, Braun J, Bopp M. Prevalence of obesity in Switzerland 1992-2007: the impact of education, income and occupational class. Obes Rev 2011; 12: 151-66.

9. Yanovski SZ, Yanovski JA. Obesity prevalence in the United States-up, down, or sideways? N Engl J Med 2011; 364: 987-9.

10. Janssen I, Shields M, Craig CL, Tremblay MS. Prevalence and secular changes in abdominal obesity in Canadian adolescents and adults, 1981 to 2007-2009. Obes Rev 2011; 12: 397-405.

11. Jia H, Lubetkin EI. The statewide burden of obesity, smoking, low income and chronic diseases in the United States. J Public Health 2009; 31: 496-505.

12. Finegood DT. Canada in context: challenging our epidemics of obesity and obesity-related chronic diseases.
Health Rep 2009; 20: 9-10.

13. Molenaar EA, Hwang SJ, Vasan RS, Grobbee DE, Meigs JB, D'Agostino RB Sr, et al. Burden and rates of treatment and control of cardiovascular disease risk factors in obesity: the Framingham Heart Study. Diabetes Care 2008; 31: 1367-72.

14. Stuart B, Lloyd J, Zhao L, Kamal-Bahl S. Obesity, disease burden, and prescription spending by communitydwelling Medicare beneficiaries. Curr Med Res Opin 2008; 24: 2377-87.

15. Singh PN. Chronic disease burden in rural India attributable to diet, obesity, and tobacco use. J Postgrad Med 2011; 57: 1-2.

16. Ministerio de Salud. Estudio de carga de enfermedad y carga atribuible, Chile 2007. Disponible en: http:// epi.minsal.cl/epi/html/invest/cargaenf2008/minuta21-07-2008.pdf. (Consultado el 18 de enero de 2012)

17. Zacarías I, Pizarro T, Rodríguez L, González D, Domper A. Programa «5 al día» para promover el consumo de verduras y frutas en Chile. Rev Chil Nutr 2006; 33 (suppl. 1): 276-80.

18. Castillo A, Smith C, Hirsch S, Brito A. ¿Es efectiva la consejería para aumentar el consumo de frutas y verduras y disminuir el riesgo cardiovascular en prevención secundaria?: una revisión. Rev Chil Nutr 2008; 35: 123-9.

19. Kain J, Leyton B, Cerda R, Vio F, Uauy R. Two-year controlled effectiveness trial of a school-based intervention to prevent obesity in Chilean children. Public Health Nutr 2009; 12: 1451-61.

20. Olivares S, Lera L, Bustos N. Etapas del cambio, beneficios y barreras en actividad física y consumo de frutas y verduras en estudiantes universitarios de Santiago de Chile. Rev Chil Nutr 2008; 35: 25-35.

21. JUNAEB. Beca de Alimentación para la Educación Superior. Disponible en:http://www.junaeb.cl/prontus_junaeb/site/artic/20100119/pags/20100119150926. html (consultado el 11 de junio de 2012).

22. Murphy, J. M. Breakfast and Learning: An Updated Review. Current Nutrition \& Food Science 2007; 3: 3-36.

23. Gajre NS, Fernández S, Balakrishna N, Vazir S. Breakfast eating habit and its influence on attention-concentration, immediate memory and school achievement. Indian Pediatr 2008; 45: 824-8.

24. Deshmukh-Taskar PR, Nicklas TA, O’Neil CE, Keast DR, Radcliffe JD, Cho S. The relationship of breakfast skipping and type of breakfast consumption with nutrient intake and weight status in children and adolescents: the National Health and Nutrition Examination Survey 1999-2006. J Am Diet Assoc 2010; 110: 869-78.

25. Goldstone AP, Prechtl de Hernández CG, Beaver JD, Muhammed K, Croese C, Bell G, et al. Fasting biases 
brain reward systems towards high-calorie foods. Eur J Neurosci 2009; 30: 1625-35.

26. Holmbäck I, Ericson U, Gullberg B, Wirfält E. A high eating frequency is associated with an overall healthy lifestyle in middle-aged men and women and reduced likelihood of general and central obesity in men. Br J Nutr 2010; 104: 1065-73.

27. Mills JP, Perry CD, Reicks M. Eating frequency is associated with energy intake but not obesity in midlife women. Obesity (Silver Spring) 2011; 19: 552-9.

28. Cho YA, Shin A, Kim J. Dietary patterns are associated with body mass index in a Korean population. J Am Diet Assoc 2011; 111: 1182-6.

29. Goldfield GS, Lumb AB, Colapinto CK. Relative Reinforcing Value of Energy-dense Snack Foods In Overweight and Obese Adults. Can J Diet Pract Res 2011; 72: 170-4.

30. Dauchet L, Montaye M, Ruidavets JB, Arveiler D, Kee F, Bingham A, et al. Association between the frequency of fruit and vegetable consumption and cardiovascular disease in male smokers and non-smokers. Eur J Clin Nutr 2010; 64: 578-86.

31. Mirmiran P, Noori N, Zavareh MB, Azizi F. Fruit and vegetable consumption and risk factors for cardiovascular disease. Metabolism 2009; 58: 460-8.

32. Crowe FL, Roddam AW, Key TJ, Appleby PN, Overvad $\mathrm{K}$, Jakobsen MU, et al. Fruit and vegetable intake and mortality from ischaemic heart disease: results from the European Prospective Investigation into Cancer and Nutrition (EPIC)-Heart study. Eur Heart J 2011; 32: 1235-43.

33. Boggs DA, Palmer JR, Wise LA, Spiegelman D, Stampfer MJ, Adams-Campbell LL, Rosenberg L. Fruit and vegetable intake in relation to risk of breast cancer in the Black Women's Health Study. Am J Epidemiol 2010; 172: 1268-79.

34. Atalah E, Urteaga C, Rebolledo A. Autopercepción del estado nutricional en adultos de Santiago. Rev Med Chile 2004; 132: 1383-8. 